\title{
Cloud Service Providers: A Comparative Analysis of Cloud Storage Pricing
}

\author{
Shahid Yaqub Tabassam \\ University of Lahore Sargodha
}

(Pakistan)

Nayyar Manzoor

University of Lahore Sargodha

(Pakistan)

\author{
Iqra Sattar \\ University of Lahore Sargodha
}

(Pakistan)

Salman Ashraf

University of Lahore Sargodha

(Pakistan)

\begin{abstract}
Cloud computing provides easy access and high performance computing on the data. Other major challenge faced by software companies is storage of data at affordable cost and makes available all the time. For both the consumers and the business customers cloud storage is a major repository. Perceptible means of contest is pricing but the difficulty of pricing plans may make a judgment difficult. In this paper the major cloud service providing companies are selected and their pricing plan is surveyed and compared them using the unit price as the means of comparison.

The pricing plan is compared by point wise for value of storage volume and an overall comparison is made by Paretodominance criteria. Under both approaches, most providers appear to offer pricing plans that are more costly and can be expelled from a procurement selection in favor of a limited number of dominant service providers.
\end{abstract}

\section{Keywords}

Cloud Storage, Cloud Computing

\section{INTRODUCTION}

Cloud storage service maintains and manages data remotely and made data available to all users over the network. In the cloud storage the data is managed by the companies that are providing the facilities. Many of these companies provide the free space up to a certain number of gigabytes. All these storage providers provide the services of drag and drop by accessing and syncing folders and files between desktops, mobile devices and the cloud storage drives.

By cloud services uses customers need not invest on storage devices. Technical support also not required for the maintenance, the storage, and backup and catastrophe recovery. The user does not need to worry about the management of the data. User does not need to know that how the data is stored and managed he has to pay and by the storage from these companies. The list of the cloud storage providers which are compared in this paper is given in the table 1.

Table 1: Cloud Storage Providers

\begin{tabular}{|l|l|}
\hline \multicolumn{1}{|c|}{ COMPANY } & \multicolumn{1}{|c|}{ WEB ADDRESS } \\
\hline Google Drive & www.google.com/drive/ \\
\hline Drop Box & www.dropbox.com \\
\hline
\end{tabular}

\begin{tabular}{|l|l|}
\hline IDrive & www.idrive.com \\
\hline SugarSync & www.sugarsync.com/en/ \\
\hline
\end{tabular}

The cloud storage in not worth when the customers store and manage data at very low cost when compared through the use of cloud. The cloud storage users can eliminate the own storage structure and rely on the cloud service providers. They may migrate to leased storage structure from own storage structure. For capital investment avoiding the cloud storage is more flexible expensive management on operational expensive only [1]. In addition if the user migrate from the own storage structure to the cloud structure it may be possible that cloud expose the lock-in phenomenon and so the price rises [2]. To avoid this situation the user has an option to use the cloud storage structure for backup only.

In both of cases the cloud storage is expanding and the need of different offers evaluation is growing as well. In present days the cloud storage providers are attracting the user to use their platform by decreasing the price day by day.

In this paper we are focusing only on the price of the cloud storage providers. The other issues like the storage work load [3], latency [6], availability [5] and bandwidth [4] can be considered. By the comparison of the price it can be decided that either to migrate or not. This comparison helps to understand the economy of scale and price structure of the industry. The structure of the paper is as follows. In the section 2 the current market size indication are provided. In section all pricing data is provided. In section 4 the pricing plan is compared. In section 5 the Pareto-dominance analysis is given.

\section{CLOUD STORAGE MARKET}

In these days the use of the cloud computing is increasing rapidly. The companies give the services to both the public cloud and the private customers. The list of the service providers is also increasing day by day.

The companies mentioned in the table 1 are the major companies that provide cloud storage services. In this paper we will analyze these companies pricing to compare them.

\section{SURVEY OF PRICE}

The survey of the companies given in the section 2 is provided here. The pricing information is taken from the websites for each service. The prices taken are correct at the time of writing. 
Many companies address the consumers and the companies separately. The consumer companies allow only for the one user and the business pricing plan is for more users.

\subsection{Google drive}

The unit price for the Google drive is calculated. The Google drive provides the $15 \mathrm{~GB}$ as free storage. The price list for the Google drive is given below [7].

The unit price for this is calculated as

Unit price $=$ monthly price $/$ space $(\mathrm{GB})$

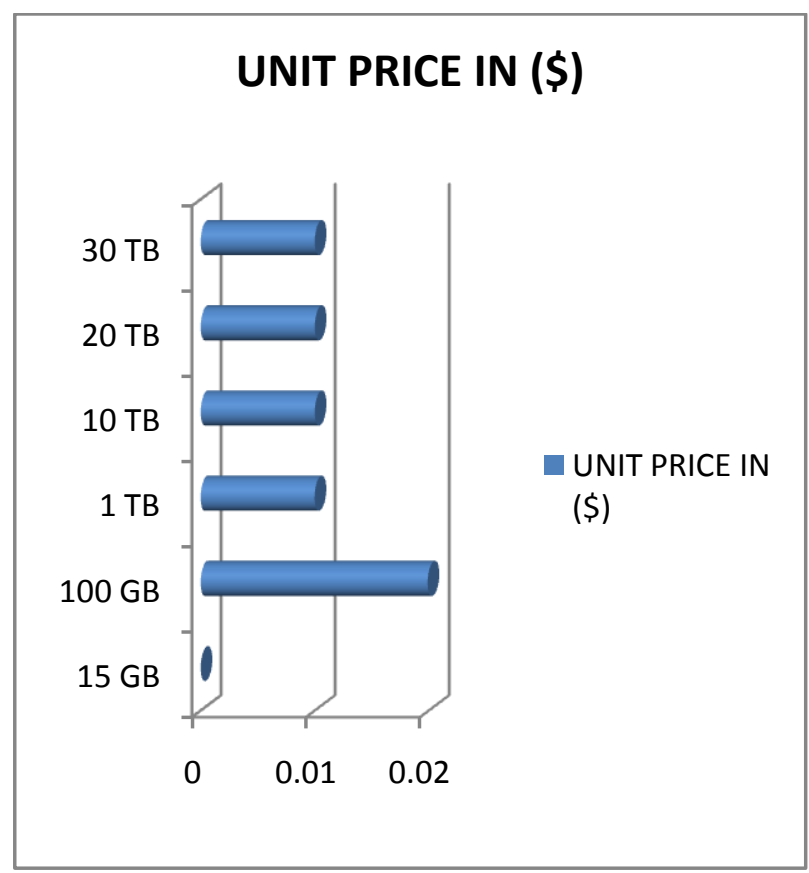

Figure 1: Unit prices for Google drive

\subsection{Drop Box}

The Drop box offers plan for both consumers and companies. It provides the free basic service to consumers and provides the free storage volume from 2-18GB.

The drop box provides the packages of $100 \mathrm{~GB}, 200 \mathrm{~GB}$ and 500GB with the price of $9.99 \$, 19.99 \$$ and 49.99 \$ per month [8].

The unit price for the drop box for consumers will be.

Table no 2 packages

\begin{tabular}{|l|l|}
\hline Total storage & Monthly price \\
\hline $15 \mathrm{~GB}$ & Free \\
\hline $100 \mathrm{~GB}$ & $\$ 1.99$ \\
\hline $1 \mathrm{~TB}$ & $\$ 9.99$ \\
\hline $10 \mathrm{~TB}$ & $\$ 99.99$ \\
\hline $20 \mathrm{~TB}$ & $\$ 199.99$ \\
\hline $30 \mathrm{~TB}$ & $\$ 299.99$ \\
\hline
\end{tabular}

\section{UNIT PRICE IN (\$)}

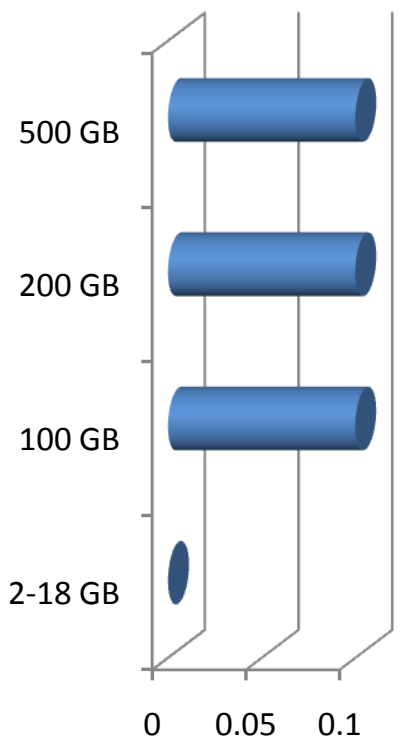

T PRICE IN

(\$)

\section{Figure 2: Unit prices for Drop Box}

\subsection{IDrive}

This also gives the services to both the consumers and the business customers. They offer the 5 GB free space and this does not required any credit card and never expires.

For personnel use it provides the $1 \mathrm{~TB}$ and the $10 \mathrm{~TB}$ packages with price of $52.12 \$$ and $374.62 \$$ per year [9]. If we convert this price for month then it will become $83 \mathrm{~GB}$ in 4.34 $\$$ and for second package 833 GB in $31.22 \$$ per month.

For business it provides the three packages $250 \mathrm{~GB}, 500 \mathrm{~GB}$ and $1.25 \mathrm{~TB}$ with price of $74.62 \$, 149.62 \$$ and $374.62 \$$ per year.

The unit price for the consumers is shown in the figure 3 . 


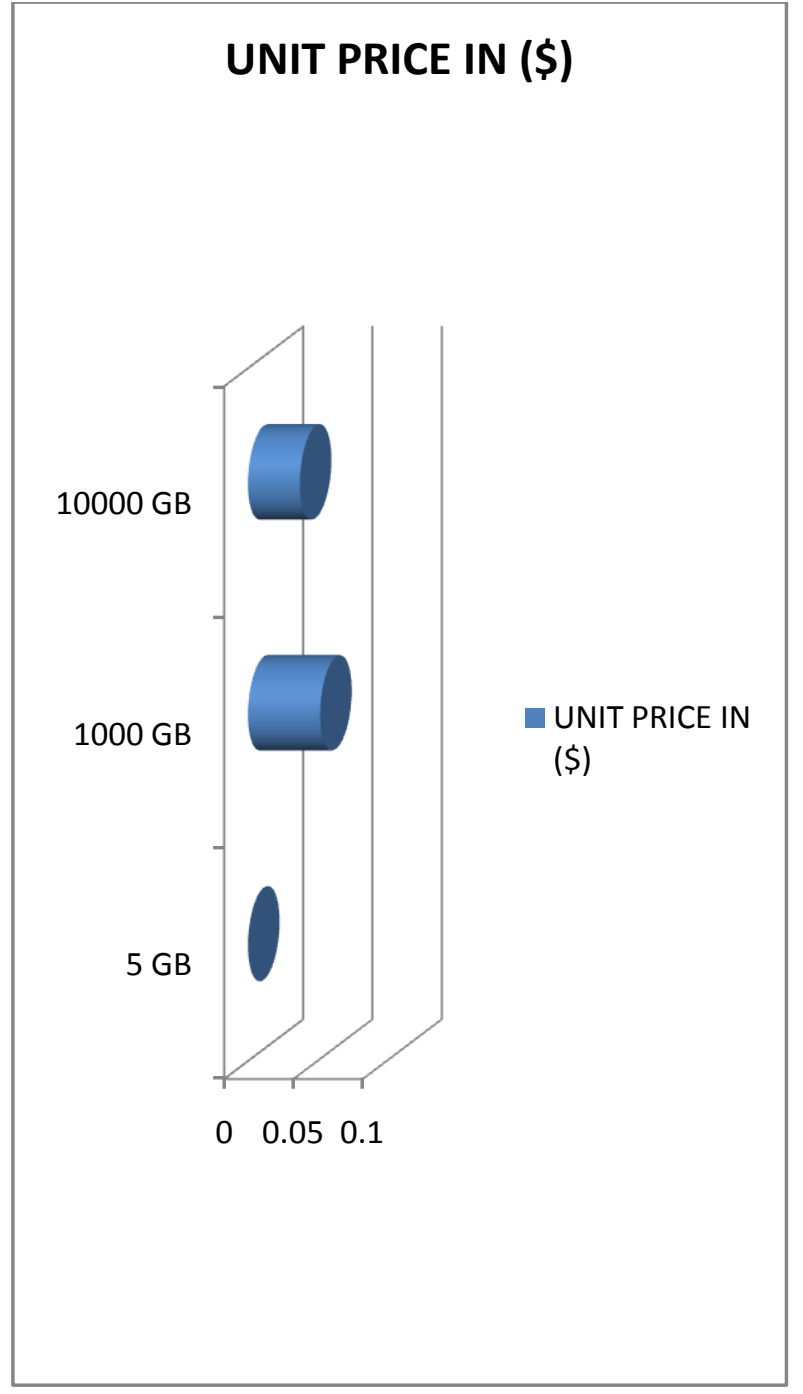

Figure 3: Unit prices for IDrive

\subsection{SugarSync}

The SugarSync provides the free volume up to the 5 GB to user on trial bases. The paid plans provided by the SugarSync are up to $500 \mathrm{~GB}$ for the consumers and it also provides the custom plan and 1TB plan for the business customers. The unit prices for the SugarSync are shown in the figure 4 .

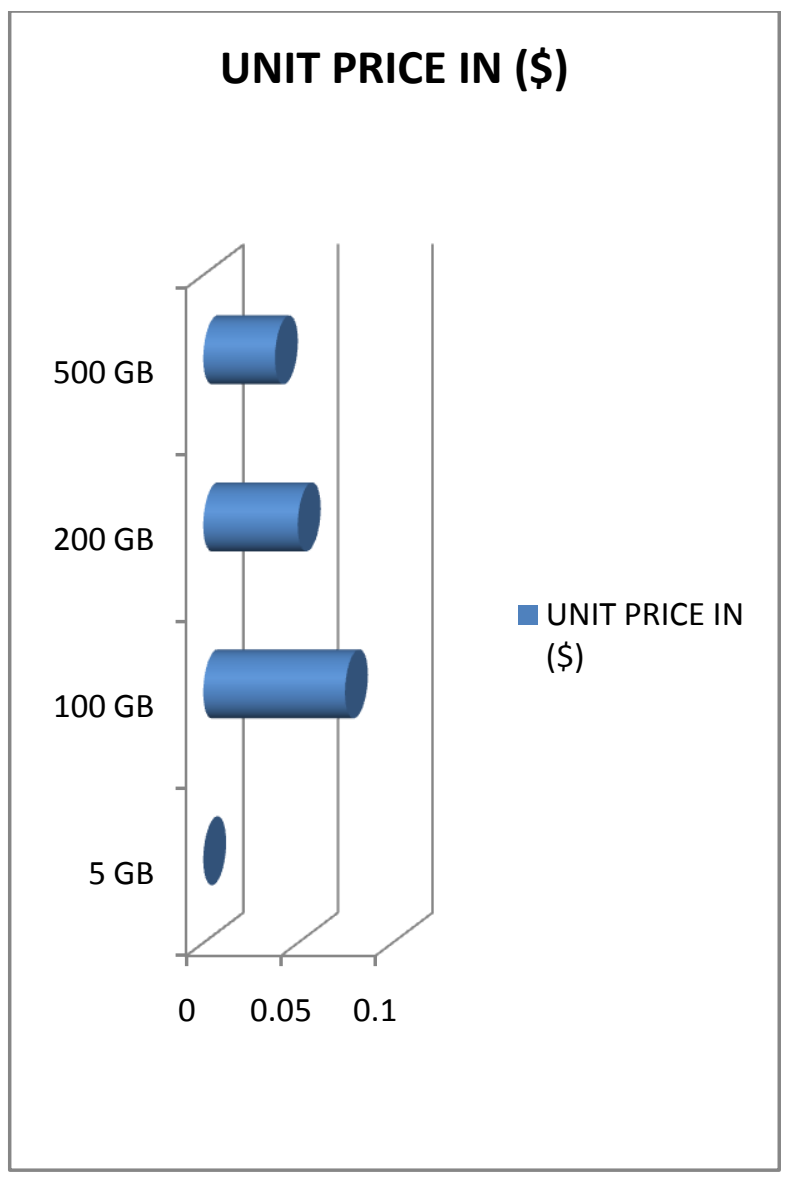

Figure 4: Unit prices for SugarSync

The business plan price for the custom package is as per requirement and for $1 \mathrm{~TB}$ package is $55 \$$ per month.

The individual plan prices are $7.49 \$ /$ month for the $100 \mathrm{~GB}$, $9.99 \$ /$ month for $200 \mathrm{~GB}$ and $18.95 \$ /$ month for $500 \mathrm{~GB}$ [10].

The amount of free space provided by these service providers is given in the table number 3 .

Table 3: Free storage comparison table

\begin{tabular}{|l|r|}
\hline SERVICE PROVIDERS & FREE SPACE \\
\hline GOOGLE DRIVE & $15 \mathrm{~GB}$ \\
\hline DROP BOX & $2-18 \mathrm{~GB}$ \\
\hline IDRIVE & $5 \mathrm{~GB}$ \\
\hline SUGARSYNC & $5 \mathrm{~GB}$ \\
\hline
\end{tabular}

The comparison of the unit prices is shown in the table 4 . This comparison is on the dominant unit prices.

Table 4: Unit prices comparison

\begin{tabular}{|c|r|}
\hline VOLUME & CONVIENIENT PROVIDER \\
\hline & \\
$0-100 \mathrm{~GB}$ & GOOGLE DRIVE \\
& IDRIVE \\
& SUGARSYNC \\
& DROPBOX \\
\hline
\end{tabular}




\begin{tabular}{|l|r|}
\hline $100-200 \mathrm{~GB}$ & \\
& GOOGLE DRIVE \\
& IDRIVE \\
& SUGARSYNC \\
& DROPBOX \\
\hline & \\
& \\
& GOOGLE DRIVE \\
& SUGARSYNC \\
& IDRIVE \\
& DROPBOX \\
\hline
\end{tabular}

\section{PARETO DOMINANCE}

In section 3 the service providers are compared as point wise basis by calculating the unit prices of each service provider. The prices plan for each of them differs from each other.

However by this we are unable to find the structural properties of the pricing plan. So to find the structural properties and classify the service providers the two part approximation is used in this section.

In the two part approximation the customer have to pay the fixed initial fee for $1^{\text {st }}$ unit plus constant price for each unit.

Fixed fee for first unit $=\mathrm{f}$

Constant fee for each unit $=\mathrm{v}$

So the overall charge payee by the customer is

$P=f+v \cdot x$

Where $\mathrm{x}$ is the volume consumption

Table 5: Parameters for Two Part Approximation

\begin{tabular}{|lcl|}
\hline Pricing Plan & $\mathrm{f}$ & $\mathrm{v}$ \\
\hline Google Drive & $1.19 \$$ & $0.02 \$$ \\
\hline Drop Box & $9.99 \$$ & $0.099 \$$ \\
\hline IDrive & $4.50 \$$ & $0.045 \$$ \\
\hline SugarSync & $9.99 \$$ & $0.099 \$$ \\
\hline
\end{tabular}

The $\mathrm{n}$ points for the resulting estimates are.

$$
\begin{aligned}
\hat{f} & =\frac{n \sum_{i} p_{i}^{(1)} / x_{i}-\sum_{i} p_{i}^{(1)} \sum_{i} 1 / x_{i}}{n \sum_{i} 1 / x_{i}^{2}-\left(\sum_{i} 1 / x_{i}\right)^{2}}, \\
\hat{v} & =\frac{\sum_{i} p_{i}^{(1)}-\hat{f} \sum_{i} 1 / x_{i}}{n} .
\end{aligned}
$$

The structure of the pricing plan can be calculated by this two part approximation. The two part approximation is attractive if the $f$ and $v$ both are low.

The price structure will be attractive if the fixed price and the marginal unit prices are low. The scatter plot shows the best pricing plans are those which are in the bottom left corner with the low fixed price and the low unit price. The worst are those which are in top right edge corner. There is a large difference in their pricing plans.

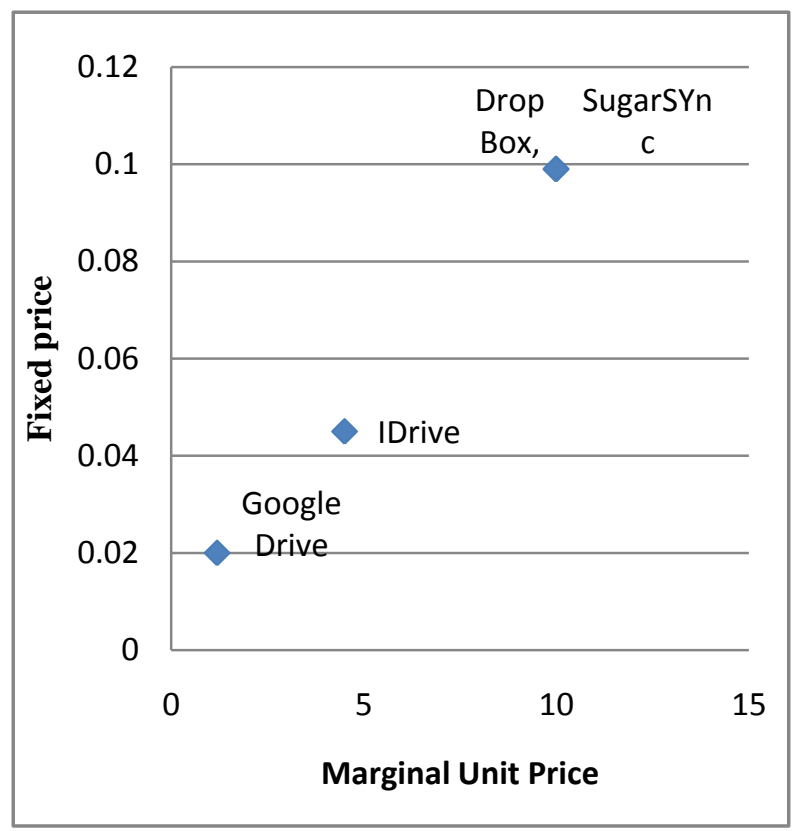

Figure 5: two part tariff parameter for consumers

The consumer pricing plans in above Figure 5 is not only a single central plan. The Google drive and the IDrive can be removed from the competition. Google Drive and IDrive offer the best pricing plans. Drop Box and SugarSync almost offer the same pricing Plan.

\section{CONCLUSION}

In this paper the cloud storage packages offered by major cloud service providers is surveyed. These package plans are compared by unit price for each of with all volume levels. We have compared the pricing plan by two methods.

By the first analysis, we concluded cheapest pricing plan. Second by applying the Pareto dominance the two-part tariff approximation identify subjugated pricing plans. A limited no. of provider is to be measured as potential providers by both analyses.

\section{REFERENCES}

[1] L. Mastroeni and M. Naldi. Storage Buy-or-Lease Decisions in Cloud Computing under Price Uncertainty. In 7th EuroNF Conference on Next Generation Internet, Kaiserslautern, 2011.

[2] Mastroeni and M. Naldi. Pricing of insurance policies against cloud storage price rises. SIGMETRICS Performance Evaluation Review, 40(2):42-45, 2012.

[3]O.A. Ben-Yehuda, M. Ben-Yehuda, A. Schuster, D. Tsafrir. Deconstructing Amazon EC2 Spot Instance Pricing. In Proceedings of the IEEE 3rd International Conference on Cloud Computing Technology and Science, CloudCom 2011, pages 304-311, Athens, Greece, November 29-December 1, 2011.

[4] I. Drago, M. Mellia, M. Munaf o, A. Sperotto, R. Sadre, A. Pras. Inside dropbox: understanding personal cloud storage services. In Proceedings of the 12th ACM SIGCOMM Conference on Internet Measurement, IMC '12, pages 481-494, Boston, MA, USA, November 14$16,2012$. 
[5] M.R. Palankar, A. Iamnitchi, M. Ripeanu, S. Garfinkel. Amazon S3 for science grids: a viable solution? In Proceedings of the 2008 international workshop on Dataaware distributed computing, DADC 08, Boston, MA, USA, June 24, 2008.

[6] M. Naldi. The availability of cloud-based services: is it living up to its promise? In Proceedings of the 9th International Conference on Design of Reliable Communication Networks DRCN 2013, Budapest, Hungary, March 4-7, 2013.
[7] https://support.google.com

[8]http://www.forbes.com/sites/amitchowdhry/2014/08/27/dro pbox-drops-price-of-1tb-storage-to-9-99-permonth/\#71de713c3bcb

[9]: https://www.idrive.com/pricing

[10]: https://www.sugarsync.com/en/pricing 